
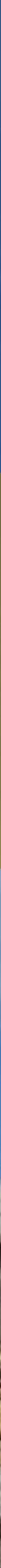


\section{Panta Rei \\ Revista Digital de Ciencia \\ y Didáctica de la Historia}

\section{6}

Revista anual

Fecha de inicio: 1995

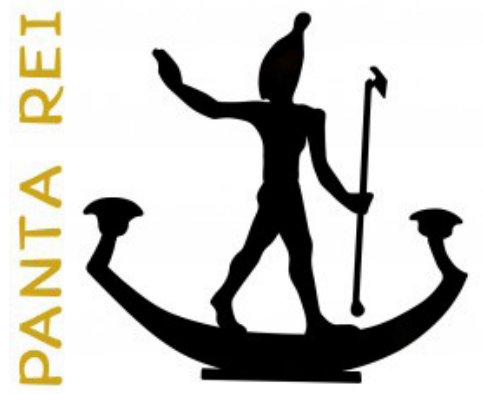

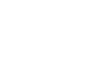




\section{CONSEJO DE REDACCIÓN}

\section{Coordinador editorial}

Egea Vivancos, Alejandro

[Didáctica de las Ciencias Sociales, UMU]

\section{Editores}

Botí Hernández, Juan Jesús

[CEPOAT, UMU]

Meseguer Gil, Antonio José

[CEPOAT, UNED]

Sáez Giménez, David Omar

[CEPOAT, UMU]

Sánchez Mondéjar, Celso Miguel

[CEPOAT, UMU]

\section{Secretaria}

Arias Ferrer, Laura

[Didáctica de las Ciencias Sociales, UMU]

\section{Responsable informático}

Martínez García, José Javier

[CEPOAT, UMU]

\section{Traducción y corrección lingüística}

Martínez Martínez, Cristina

[Sociedad Española de Lenguas Modernas]

Albaladejo Albaladejo, Sara

[ISEN, UMU]

\section{CONSEJO ASESOR}

Albero Muñoz, M. ${ }^{a}$ del Mar

[H. ${ }^{a}$ del Arte, UMU]

Chapman, Arthur

[History Education, UCL, Reino Unido]

Cobacho López, Ángel

[Derecho, UMU]

Egea Bruno, Pedro M. ${ }^{\text {a }}$

[Historia Contemporánea, UMU]

García Atienzar, Gabriel

[Prehistoria, UA]

González Monfort, Neus

[Didáctica de las Ciencias Sociales, UAB]

Haber Uriarte, María

[Prehistoria, UMU]

Hutson, Scott R.

[Anthropology, UK, EEUU]

Irigoyen López, Antonio

[Historia Moderna, UMU]

Mahony, Simon

[Digital Humanities, UCL, Reino Unido]

Marsilla de Pascual, Francisco Reyes

[Técnicas historiográficas, UMU]

Miralles Maldonado, José Carlos

[Filología Clásica, UMU]

Molina Gómez, José Antonio

[Historia Antigua, UMU]

Noguera Celdrán, José Miguel

[Arqueología, UMU]

Pérez Molina, Miguel Emilio

[Filología Clásica, UMU]

Prados Martínez, Fernando

[Arqueología, UA]

Sánchez Ibáñez, Raquel

[Didáctica de las Ciencias Sociales, UMU]

Sancho Gómez, Miguel Pablo

[Educación, UCAM]

Vilar García, María José

[Historia Contemporánea, UMU]

Zamora López, José Ángel

[Próximo Oriente Antiguo, CCHS-CSIC] 

Artículos

Los orígenes de la tecnología a debate: una revisión de las primeras industrias líticas.

Arturo Cueva Temprana.

De arqueología menorquina: Maria Lluïsa Serra Belabre y los círculos talayóticos de Sant Vicenç d' Alcaidús (Alaior, Menorca).

Octavio Torres Gomariz.

Nuevas cuestiones sobre el anfiteatro de Zaragoza.

José David Mendoza Álvarez.

Castidad o castigo. El estupro de las Vestales como símbolo de desorden social en Roma.

Juan Antonio Montalbán Carmona.

La Historia antigua en la Biblioteca de Focio.

Juan Luis Posadas Sánchez.

Usos sociales de la historia. La estrategia de Olga Cossettini, Rosario, 1935-1943.

Paula Caldo, Micaela Pellegrini Malpiedi y Agustina Mosso

Contribuciones a la didáctica de la Historia a través del método de análisis del objeto: como ejemplo... una "vasulla".

Nayra Llonch Molina y Verónica Parisi Moreno.

How are digital methods changing research in the study of the classical world? An EpiDoc case study. Katherine Steiner y Simon Mahony.

\section{Reseñas}

I Congreso Internacional "Creando ciudadanos, construyendo identidades. El uso del patrimonio material e inmaterial en la enseñanza de la historia."

José Díaz Serrano, Ainoa Escribano Miralles, Ana Isabel Ponce Gea y David Verdú González 151

Beckert, S. (2014). Empire of cotton: A global history. New York: Alfred A Knopf. 640 págs.

Ricky D. Mullins Jr..

Coumert, M. y Dumézil, B. (2013): Los reinos bárbaros en Occidente (traducción de Peinado Santaella, R. G.: Les royaumes barbares en Occident, Presses Universitaires de France, 2010). Editorial Universidad de Granada. Granada. 156 págs.

José Ángel Castillo Lozano.

La prehistoria en Las tres edades de Buster Keaton.

Alberto Lombo Montañés y Esther Rodríguez Ortiz.

Normas de publicación/Publishing rules 



\title{
Usos sociales de la historia. La estrategia de Olga Cossettini, Rosario, 1935-1943
}

Social uses of history. The strategy of Olga Cossettini, Rosario, 19351943

\author{
Paula Caldo ${ }^{1}$ \\ Consejo Nacional de Investigaciones Científicas y Técnicas \\ Universidad Nacional de Rosario \\ Micaela Pellegrini Malpiedi² \\ Consejo Nacional de Investigaciones Científicas y Técnicas \\ Universidad Nacional de Rosario \\ Agustina Mosso ${ }^{3}$ \\ Universidad Nacional de Rosario
}

Recibido: 18/01/2016

Aceptado: 07/04/2016

Para citar este artículo: Caldo, P., Pellegrini Malpiedi, M., y Mosso, A. (2016). Usos sociales de la historia. La estrategia de Olga Cossettini, Rosario, 1935-1943. Panta Rei. Revista Digital de Ciencia y Didáctica de la Historia, 97-110.

ISSNe: 2386-8864

DOI: $10.6018 /$ pantarei/2016/6

\section{Resumen}

Este artículo propone pensar algunos de los usos sociales de la historia. Justamente, recupera una experiencia pedagógica que asumió el desafío de educar a los niños de cara a la vida. En el año 1935, Olga Cossettini es nombrada directora de la Escuela Carrasco, institución a la que se le asigna carácter experimental. Ella elaboró entonces un Plan Asociado en el cual el contenido curricular incorporó las características del contexto. La decisión estuvo fundada en elementos pedagógicos dando como resultado un modo de enseñar y aprender historia mirado desde abajo.

\section{Palabras Clave}

Ciencias Sociales, Experiencia Educacional, Aprendizaje Histórico, Didáctica de la Historia.

\footnotetext{
Abstract

This article is focused on social uses of history. It recovers a pedagogical experience that adopted the challenge of educating children for facing life. In 1935, Olga Cossettini was named

1 Para contactar con esta autora: Paula Caldo. Consejo Nacional de Investigaciones Científicas - Universidad Nacional de Rosario. paulacaldo@gmail.com.

2 Para contactar con esta autora: Micaela Pellegrini Malpiedi. Consejo Nacional de Investigaciones Científicas - Universidad Nacional de Rosario. micaelapellegrini89@gmail.com.

3 Para contactar con esta autora: Agustina Mosso. Universidad Nacional de Rosario. agustina_mosso@ hotmail.com
} 
director of Carrasco School, an institution with an experimental character. Then, she developed an Associated Plan in which curricular content involved several context features. The decision was based on pedagogical elements, giving as a result a particular way of teaching and learning history from below.

\section{Keywords}

Social Sciences, Educational Experience, History Instruction, Didacticism.

\section{Introducción}

El presente artículo está motivado en una preocupación que nos ha inquietado en los últimos tiempos: los usos sociales de la historia. Esto es, más allá de los lugares específicos donde los historiadores e historiadoras nos reunimos a debatir y reflexionar sobre las formas de construir conocimiento sobre el pasado, existen muchos otros ámbitos donde la historia cristaliza como un saber que ata los sentidos de las prácticas. Suponemos que, es aquí donde, al tiempo que el saber histórico comienza a ser utilizado por la gente, se aleja de los historiadores adquiriendo una autonomía basada en la veracidad fáctica del pasado (Caldo y Garcilazo, 2015; Guha, 2016; Pereyra, 1997). Tal autonomía lo transforma en términos no necesariamente próximos a las discusiones epistemológicas en torno al conocimiento histórico, generando así sugerentes alteraciones que son, justamente, las que prenden en el sentido común y ayudan a los ciudadanos y ciudadanas a tramitar los avatares de la vida cotidiana.

Empero, esta forma de entender y pensar la historia no es ingenua, por el contrario, esta construida generalmente sobre los principios de la historiografía tradicional. Peter Burke lo afirma: "también podríamos darle a este paradigma el nombre de visión de sentido común de la historia, aunque no para elogiarla, sino para recalcar que a menudo se ha supuesto que era la manera de hacer historia y no se consideraba una forma más de abordar el pasado" (2003, p. 15). Esto es, la historia narra los hechos del pasado "tal cual fueron". Los documentos tamizados por la crítica son el certificado de veracidad de ese relato convertido en certeza. Aunque para la escucha de quienes discutimos sobre teorías de la historia estas afirmaciones parezcan obsoletas, no lo son cuando comenzamos a transitar por fuera de los ámbitos académicos. Por lo tanto, y encaminándonos a presentar nuestro caso, nos aventuramos a aseverar que la escuela pública tiene mucho que ver con esos enraizamientos sociales. A los efectos de pesquisar estos últimos, quienes oficiamos de autoras del presente artículo, elaboramos el Proyecto de Investigación y Desarrollo (PID) "La historia en el currículum de las escuelas primarias en la provincia de Santa Fe 1880-1930", 1HUM437 Resolución CS No 073/2014, Facultad de Humanidades y Artes - Universidad Nacional de Rosario. En el mismo preguntamos por qué y qué historia se enseña en estas instituciones de reparto masivo y obligatorio de saberes. Porque sin dudas, la escuela fue y es el espacio por excelencia de distribución masiva del conocimiento en general y del histórico en particular. Así que nos propusimos interrogar algunos aspectos de lo acontecido con el reparto de los saberes históricos en este lugar tan particular de la cultura. Esta investigación, proyectada desde la historia socio-cultural (Chartier, 1999, 2005; Eley, 2008), detiene su mirada en las cinco primeras décadas de existencia del sistema educativo argentino con la intención de discutir un supuesto particular. Esto es, la efectiva concreción del enfoque de corte nacional que, por entonces, tuvieron los contenidos curriculares estipulados para la enseñanza de la Historia en las escuelas primarias, a partir del año 1884. Año en que fue sancionada la Ley de Educación Común $\mathrm{N}^{0} 1420^{4}$. La misma poseía un texto

4 La Ley de Educación Común № 1420 fue sancionada durante el gobierno de Julio Argentino Roca. Este tuvo a bien presidir una gestión abocada a legislar, en clave preferentemente liberal, diferentes aspectos del orden social. Fue un gobierno de paz y administración, donde fueron ordenadas muchas instituciones bajo un claro control estatal: la sistematización de las escuelas primarias y la creación de Registros Civiles, son 
detallista que prescribió sobre todas las dimensiones del sistema educativo, incluidos los contenidos curriculares. Eran los tiempos de consolidación del Estado nacional centralizado (Ansaldi, 1989) y, en este marco, la escuela primaria, pública, obligatoria, laica y gratuita operó como un aglutinador de masas exclusivo y, en cierto sentido, exitoso. De tal forma, las prescripciones curriculares expusieron un relato nacional homogéneo que desconoció las particularidades regionales de la Argentina (con sus diferencias no solo provinciales sino regionales) (Cucuzza, 2007; Finocchio, 2013; Romero, 2004). No obstante, nuestro Proyecto de Investigación y Desarrollo antes citado, habilita a sospechar de ese supuesto porque pondera la tensión entre las prescripciones y las prácticas. Es decir, más allá de la direccionalidad marcada por la letra escrita, entendimos que en las prácticas situadas podían emerger diferencias que, discutiendo las directivas nacionales, dieran lugar a experiencias educativas alternativas, novedosas y capaces de recuperar historias, relatos, registros y expectativas de carácter regional/local (con su medio, su gente, su historia y sus formas de apropiarse del mundo). Casualmente, la historia de la educación ha dado sugerentes balances que permiten situar la decisión teórica y metodológica tomada en este artículo. Hoy día la historia de la escuela y de la escolaridad está siendo también auscultada en la clave de "la cultura" tanto en sus aspectos simbólicos como materiales. Así, tomar como objeto de estudio la escuela en tanto lugar habitado por actores cuyas prácticas se nutren y/o generan de símbolos, rituales, léxicos pero también elementos materiales, es una entrada para enriquecer las formas de historiar la educación escolarizada pero también genera la necesidad de renovar las fuentes: cuadernos de clase, notas de docentes, epistolarios, imágenes, etc. (González Delgado, 2013; Viñao Frago, 2008).

Inspiradas en las preocupaciones teóricas arriba citadas fue que descubrimos la labor pedagógica de las hermanas Olga y Leticia Cossettini. Una tarea ubicada temporalmente entre 1930-1950. Ambas mujeres, formadas en escuelas normales, tuvieron la posibilidad de ejercer la docencia en distintas instituciones educativas y ocupar diferentes cargos relativos a la educación escolar. Fundamentalmente Olga se inició como maestra pero rápidamente pasó a ser directora, primero del Departamento de Aplicación de la Escuela Normal de la ciudad de Rafaela (Santa Fe) y luego de la escuela Dr. Gabriel Carrasco (en Rosario-Santa Fe).

Los nombres de estas maestras quedaron grabados en la historia de la educación argentina justamente por ser impulsoras de los postulados del escolanovismo (Carli, 2003; Colotta, 2002; Ossanna, 1993; Pelanda, 1995). Una forma de entender la enseñanza alumbrada a fines del siglo XIX en Europa y, desde entonces, proyectada en distintas realidades geográficas a lo largo de la primera mitad del siglo XX (Caruso, 2001). La novedad de la Escuela Nueva fue la puesta en el centro de la escena educativa del niño, con sus modos de aprender, con las actividades y con plena confianza en su desarrollo cognitivo. En la experiencia Cossettini esto cristalizó en una escuela pública, estatal y periférica, por lo cual supusimos que la originalidad de la apuesta lejos de residir en los contenidos a enseñar, lo hizo en el tratamiento didáctico de los mismos. No obstante, no fue así. En el mundo de las prácticas de la enseñanza, muchas veces, la forma didáctica transforma al contenido. Esto es, cuando Olga, Leticia y las maestras de la escuela Carrasco deciden trabajar a partir de las posibilidades de aprendizaje de los niños, necesariamente fueron transformando el contenido de la enseñanza. Es justamente este modo de enfocar el contenido a enseñar, el hallazgo de esta investigación. Resulta útil para pensar un estilo particular de enseñar las ciencias sociales en general y la historia en particular situando como disparador a la geografía. No obstante, y aquí nuestra hipótesis a demostrar, al introducirnos en la descripción de la propuesta Cossettini advertimos cómo se fue transformando el contenido a transmitir en base a la didáctica y las teorías del aprendizaje y no así en las discusiones teóricas en torno a la producción de conocimiento disciplinar. Por lo cual, se innova en las formas más no así en la concepción acerca del conocimiento histórico.

Ahora bien, ingresamos a "la caja negra" (González Delgado, 2013) de las hermanas

algunos ejemplos de ello. Para ampliar información sobre el tema pueden consultarse, por ejemplo, el clásico libro de Botana (1994) o González Bernaldo de Quirós (2014) o también Lobato (2010), entre otros. 
Cossettini, por el minucioso trabajo de resguardo que se realizó con el material. Esto es, desde los comienzos de sus prácticas, estas maestras tuvieron el cuidado de hacer de la experiencia una colección que cuidaron ellas primero y sus exalumnos y familiares, luego. Por lo cual, desde el año 2001 las hermanas Cossettini tienen editados sus escritos bajo el título Obras completas, con sello editorial de AMSAFE (Asociación del Magisterio de Santa Fe) y, a su vez, desde el año 2006, la ciudad de Rosario (Santa Fe - Argentina) cuenta con el Archivo Pedagógico Cossettini, donde están reunidos cuadernos de alumnos, diarios de clase escritos por las maestras, epistolario, colecciones de revistas, planes de estudio, la biblioteca personal y demás papeles elaborados en relación a la experiencia educativa de estas hermanas. Así, las afirmaciones sostenidas a largo de este artículo cuentan con estos respaldos heurísticos.

Finalmente y a modo de síntesis, el caso Cossettini es un pretexto para, por un lado, abrir una línea de discusión alrededor de la construcción epistémica de los usos sociales de la historia y, por otro, abordar la tensión historia nacional - historia local en los contenidos curriculares. Para ello el artículo se estructura en tres partes. Una introducción seguida de un desarrollo divido en tres apartados. El primero sitúa a las maestras, el segundo abre los lineamientos pedagógicos generales de la propuesta y el último se detiene en la articulación de la historia dentro del Plan Asociado de las hermanas Cossettini. Por último, cerramos con unas palabras de síntesis explicativa.

\section{Aproximaciones a la experiencia Cossettini}

Siguiendo el atajo biográfico recuperado por la historiografía del presente decimos (Bolufer, 2008; del Pozo Andrés, 2013): Olga y Leticia fueron dos maestras que, pese a estar formadas en el interior del proyecto normalista de principios de siglo XX, capitalizaron los aportes del escolanovismo en sus prácticas y en sus pensamientos en torno a la tarea de educar. Las hermanas Cossettini nacieron en San Jorge, un municipio del centro-oeste de Santa Fe. La primera, en 1898, la segunda, en 1904. Ambas siguieron la profesión del padre, Antonio Cossettini: el magisterio. En 1914 y con sus 16 años, Olga, la mayor, egresó de la Escuela Normal de Maestros Rurales de Coronda. Una vez alcanzado el título trabajó en distintas localidades de la provincia hasta llegar a Rafaela, la próspera ciudad del oeste santafesino, paradigma de la colonización del siglo XIX. Allí comenzó a ejercer en la misma institución en la que su hermana Leticia se graduaría de maestra. La experiencia acumulada en el Normal "Domingo de Oro" fue central en la historia de las hermanas Cossettini. Entre los años 1930 y 1935, Olga fue directora del Departamento de Aplicación del Normal de Rafaela. Por aquellos días la directora general del establecimiento educativo era Amanda Arias (1894-1957), una destacada maestra que supo orientarla por la senda del escolanovismo (Fernández y Caldo, 2013).

Abrimos un paréntesis para aclarar que el contexto político provincial permitió que germinara el escolanovismo en tiempos donde las libertades políticas se clausuraban en el orden nacional. Justamente, en 1930 la sociedad argentina experimentó el primer golpe de Estado que situó al General José Félix Uriburu en la presidencia de la nación. El mandato de Uriburu (1930-1932) al tiempo que cerraba la experiencia democrática de los gobiernos radicales (entre 1916 y 1930: dos periodos de Hipólito Yrigoyen mediados por uno de Marcelo T. de Alvear), abría un momento para la contracción democrática y el retorno de prácticas conservadoras declaradamente católicas. Sin embargo, en Santa Fe, una provincia que había sido intervenida por la gestión radical, se preparaban para los comicios que darían la victoria, en el año 1931, al candidato del Partido Demócrata Progresista, Luciano Molinas (1931-1935). Desafiando los lineamientos políticos de la época, Molinas esbozó como eje principal de su campaña los postulados de la Constitución santafesina de 1921. Una Carta Magna de raigambre liberal, anticatólica y democrática. Reeditar aquellos principios obligó a la nueva administración a emprender distintos proyectos que derivaron en la Constitución provincial de 1933 y en la consecuente Ley de Educación № 2364 del año 1934. De este modo, las políticas educativas, ahora sí operarían en clave de gratuidad, obligatoriedad, universalidad y laicidad. En esta dirección, se dispusieron las bases de una política educativa 
descentralizadora donde los Consejos Escolares de Distrito serían piezas centrales, disputando espacios de intervención, financiamiento y gestión escolar al Director General de Escuelas. Los nuevos Consejos Escolares fueron investidos de atributos electivos y de una autonomía inédita. En este nuevo contexto, contarían con rentas propias y con la capacidad para generar tributos destinados a fortalecer la educación provincial, como así también para nombrar y controlar tanto a los maestros como a los funcionarios administrativos del ámbito educacional. Pero el aire reformista no solamente envolvía aspectos de raíz administrativa, sino también otros de carácter metodológicodidáctico. En este sentido, es clave destacar la importancia adjudicada a la aplicación de métodos activos para la enseñanza, como también el valor asignado a las prácticas pedagógicas de matriz estética, recreativa y deportiva. Si Santa Fe era la nota singular en medio de la trama sociopolítica y cultural de un país donde los rasgos democráticos y participativos eran corroídos, no resulta extraño que en esta provincia en particular, surgieran experiencias como la de Olga en la Escuela Normal "Domingo de Oro" de Rafaela (Pérez, 2000; Videla, 2006).

Sin dudas, las intervenciones de Olga en Rafaela fueron, parafraseando el título de su libro del año 1935, el primer ensayo de Escuela Serena en las colonias santafesinas. La expresión "Escuela Serena" fue elegida para nominar las distintas experiencias pedagógicas implementadas por las hermanas Cossettini. El nombre, lejos de ser azaroso, se debe a la obra de quien Olga considera su maestro: el pedagogo italiano José Lombardo Radice. Justamente, en Italia con el título Escuela Serena se distinguieron los establecimientos educativos que seguían los lineamientos pedagógicos de Radice. El pedagogo es una cita obligada en los escritos de las hermanas Cossettini al momento de definir qué entienden por educación, cómo conciben la enseñanza, cómo piensan el rol del alumno (Cossettini y Cossettini, 2001).

En 1935 Olga y Leticia reciben el traslado a Rosario. En esta localidad, fueron designadas para el desempeño de sus funciones docentes en la escuela de gestión pública "Dr. Gabriel Carrasco", situada en el barrio Alberdi, que había sido incorporado al municipio rosarino hacía poco más de dos décadas. Al respecto Olga dirá:

Nuestra escuela está ubicada en el límite de la ciudad y el campo. El ruido que nos envía la ciudad por su camino central, brazo de unión con el norte santafesino, ruido incesante de motores en marcha, nos llega amortiguado, como nos llega adormecido el paso de las dragas y lanchones que surcan el río vecino. Pero la brisa de ese río amigo, el verdor de sus barrancas, el canto de los pájaros, nos traen armonías todas las mañanas y los niños que bajan de los ranchos, de las casitas obreras y de las viviendas mejores, pueblan la escuela de bullicio hasta el sol de la tarde.

(Cossettini y Cossettini, 2001)

Olga en la dirección, y Leticia, junto a otras docentes, desde las aulas, diseñaron los lineamientos de una nueva edición de La Escuela Serena santafesina, la versión rosarina que vendría a prolongar a la ensayada en Rafaela.

El espesor de la cultura rosarina de la época permitió que las hermanas Cossettini no solamente continuaran su trayecto en el campo de la docencia, sino que comenzaran a crecer con vuelo propio. Esos logros personales vinieron rápido puesto que, casi cuando finalizaba el año 1935, el Director General de Escuelas, Dr. Pío Pandolfo, dictó un decreto mediante el cual distinguía a la escuela dirigida por Olga con el calificativo de "experimental". Así quedaba legalizada la autonomía didáctica de la directora para decidir sobre qué y cómo enseñar. Al respecto, el Ministro de Instrucción Pública y Fomento, Juan Mantovani, dirá:

Eso es lo que ocurre en la Escuela Experimental 'Dr. Gabriel Carrasco', instalada en el barrio Alberdi de la ciudad de Rosario. Se realiza en ella, bajo la experta dirección de Olga Cossettini, educadora de un don excepcional para la comprensión y tratamiento de los niños, un ensayo del más alto interés pedagógico. Allí se cumplen los programas de la escuela primaria común, pero se aplica una organización del trabajo escolar y procedimientos didácticos apoyados en los más hondos resortes psicológicos del niño, 
particularmente en la libre expresión de su quimérico mundo interior y de su fértil y animada fantasía.

(Cossettini y Cossettini, 2001, p. 183-184)

En estas condiciones la escuela funcionó hasta el año 1944. Desde entonces vendrían tiempos difíciles, el cuarenta y cuatro trajo el final de la escuela experimental y el cincuenta la cesantía de las protagonistas, Olga y Leticia. Ya separadas de sus cargos docentes, siguieron trabajando por la educación nacional y provincial, difundiendo sus ideas en tareas de gestión y en trabajos editoriales.

Ellas cuestionaron las formas de educar tradicionales valiéndose del discurso de la pedagogía escolanovista. El movimiento de la escuela nueva discutió las bases didácticas sobre las cuales se había constituido el sistema educativo en general y la formación docente en particular. Asumirse escolanovista implicaba una apuesta por el reconocimiento de los intereses y de las formas de expresarse de los niños. En esta clave, los docentes dejaron de ser los portavoces del saber para diseñar una compleja trama de actividades que permitía a los estudiantes aprender haciendo, descubriendo, leyendo el mundo, exteriorizando dotes creativas de estricto orden interno. En consecuencia, si todo atisbo de debate político en términos partidarios quedaba excluido de las aulas, la política, el desacuerdo se instalaba en el plano de la interpretación de la tarea docente.

Ahora bien, en medio de las disputas y transformaciones sugeridas por las hermanas Cossettini ¿qué pasó con la enseñanza de la historia?, ¿fue transformada por aquella forma innovadora o se mantuvo ligada a los contenidos tradicionales?

\subsection{Pensar lo social desde el lugar, una propuesta interdisciplinaria}

Si nos preguntamos qué lugar ocupó la historia en la escuela de la señorita Olga, como primera cuestión tenemos que definir qué se entendió por educación en el marco de este proyecto. Para el conjunto de maestras que llevaron adelante esta experiencia, educación "es la compenetración de las almas, esto es, un estado de conciencia en el cual el maestro desaparece como individualidad distinta a la de los alumnos y se funde en su momento espiritual, viviéndolo como suyo para impulsar hacia aquellas posiciones más altas por él ya alcanzadas" (Cossettini y Cossettini, 2001, p. 16).

Así, el maestro conforma una amalgama con sus alumnos y juntos se proyectan hacia la experiencia de aprender la realidad que los rodea. Una propuesta que respeta la capacidad cognitiva del alumno y opera a partir de ella y con ellas y sobre las cosas que los chicos saben y conocen, buscando entenderlas para luego ir por más saberes.

De este modo, cuando Olga reflexiona sobre la enseñanza de las ciencias en general y de la historia en particular, sitúan como alma mater de la propuesta a la geografía. Lo hace porque para ella el paisaje (el medioambiente) es lo cercano, lo que los niños y las niñas que acuden a la escuela tienen más a mano para activar el aprendizaje. Esta disciplina será el trampolín que permita a los pequeños lanzarse al estudio de las otras ciencias, entre las que la historia ocupa un lugar que oscila entre planteos locales (la historia del barrio) y otros de corte nacional (el calendario de efemérides oficiales). La historia de la humanidad es descubierta por los niños:

...en los hombres humildes que amasaron el progreso de este pueblo. Ellos saben contar mejor que el maestro su historia. Hay honda emoción en estos simples relatos. Los niños escuchan felices, perciben la lucha del hombre que se hace fuerte en la adversidad, tiene la intuición de que el progreso es producto del esfuerzo y del sacrificio diario, y cuando escriben sus impresiones respetan el lenguaje y apuntan entre comillas sus expresiones típicas (a veces en dialecto), porque la gramática, esa gramática que tanto preocupa a los pseudos maestros, está viva en la lengua que se enriquece cuando su espíritu penetra en los otros por obra de amor y simpatía.

(Cossettini y Cossettini, 2001, p. 53) 
Así, los estudiantes se acercan a la historia a partir de los relatos de los vecinos. A estos protagonistas directos los sitúan en el espacio (llamado geografía) y a partir de allí van tejiendo sus saberes, su pasado, su lengua..., logrando un conocimiento integral del barrio que se proyecta, en lo que Olga Cossettini llama, el instinto geográfico. Un instinto que si bien es adquirido en el lugar, resulta clave para conocer, comprometerse, querer y cuidar el espacio que nos rodea y de ahí lanzarse a conocer y sentir esos mismos valores por otras tierras y realidades desconocidas y lejanas.

Esta invitación a conocer el pasado a partir de los relatos de los vecinos, nos recuerda a Natalie Zemon Davis (1998) preguntándose: ¿quién es el dueño de la historia? La citada historiadora, a los efectos de responder revisa las argumentaciones de los posibles propietarios: los historiadores, los profesores, los pueblos (grupos, minorías, etc.), la cultura occidental. Ella ensaya en términos reflexivos las distintas respuestas y, finalmente, concluye diciendo que la historia es una herencia colectiva, que debemos trabajar para obtener, cada uno, desde su lugar. Asumiendo las distancias teóricas, Cossettini establece el pasado (ella dice la historia) como herencia colectiva, y sitúa a los niños en el lugar de la búsqueda para elaborar ese pasado. Por eso, en las clases de historia van a conocer el barrio, lo recorren, lo observan, lo dibujan y, en simultáneo, van entrevistando a los vecinos para aprender cómo viven y vivieron ese lugar, esas prácticas, esas instituciones, esos servicios, ese paisaje, ese río.

Sin dudas, esta propuesta educativa particular estuvo organizada a partir de los problemas del aprendizaje (cómo aprenden los niños) y no desde la lógica del saber disciplinar (la historia). Las maestras, lejos de exponer teorías, cumplían funciones de apoyatura, la guía al estilo del preceptor sugerido por Jean-Jacques Rousseau (1985), de estímulo para que el trabajo se realice en la dirección esperada.

Al preguntarnos qué historia enseñaban estas docentes, emerge como respuesta el principio de solidaridad humana, que primaba en las aulas de las hermanas y preparaba para la vida social. En los escritos consultados se traslucía la necesidad constante de enseñar al individuo los problemas del municipio, pero en la generalización del sentimiento de solidaridad. La historia, circunscrita dentro de las ciencias sociales junto con geografía e instrucción cívica, la economía, ayudaba a formar conciencia social a través de la educación en hábitos, capacidad, conducta, aptitud. El hombre situado en el medio era, en la Escuela Carrasco, el punto central de la enseñanza de las ciencias sociales, seguía además siempre el proceso de su transcurso por la vida, enmarcado en sus descubrimientos y en sus luchas. Y para alimentar el tratamiento de ese eje, lejos de fragmentar el conocimiento científico, se lo abordó en términos relacionales. Como en la vida misma, donde los principios, reglas y conceptos lejos de operar en secuencias lógicas y ordenadas se mezclan y producen así los efectos de la cadencia de la vida. La participación de los educadores estaba delimitada; intervenían solo cuando los alumnos lo solicitaban, aunque los interesaban para ir a la biblioteca, facilitadora de abundante material de lectura, si bien de igual forma eran aceptados los libros traídos desde los hogares (Cossettini y Cossettini, 2001).

Los maestros promovían y guiaban a los diferentes grupos. Por ejemplo, las preguntas del maestro estaban presentes para que la memoria de los alumnos fluyera a los efectos de recordar experiencias, visitas, charlas vividas. Asimismo, el cuidado del orden, la prolijidad y las normas de convivencia eran tema recurrente de trabajo cotidiano. El maestro tomaba lo que el niño ofrecía y lo tamizaba para ofrecerlo nuevamente. En el contexto de los estudios sociales, la lectura era ejercicio de pensamiento y de palabra, y entraba en juego en todos los momentos de la clase. Poseían la misma importancia tanto la lectura en voz alta como la silenciosa.

Ahora bien, ya en la primera mitad del siglo XX, los beneficios de comenzar el estudio del pasado por los aspectos más cercanos a la realidad del niño fueron enfatizados por pedagogos y psicólogos educacionales (de Amézola, 2008). Justamente, para el caso de la propuesta Cossettini, partir de la dimensión espacial generaba la posibilidad de reconocer y aprehender el medio ambiente, primero en una dimensión local (con la ponderación de sus problemas en un cruce de análisis sociohistórico) y luego avanzar rumbo a una nacional y también universal. Con este método las hermanas 
lograban que sus alumnos comprendieran mejor el pasado; ya que al empezar con el contexto más próximo el aprendizaje se proyectaba de lo simple y cercano a lo complejo y lejano.

Las docentes estimulaban la inquietud de hablar y escribir sobre algo visto e intuido al observar. Observaciones personales, descripciones, conversaciones sostenidas con la maestra, el compañero, el albañil, el agricultor, el pescador sobre los temas estudiados en el año. Los chicos leían, investigaban. Campesinos, obreros, pescadores, albañiles, y vecinos en general, oficiaron de maestros en aquellas ocasiones en que los niños de la Escuela Carrasco los visitaban con el fin de aprender. Mientras que los primeros narraban o mostraban sus saberes en la cadencia del hacer, los pequeños aprendices anotaban en sus cuadernos las sensaciones despertadas en la escucha y la observación. Estudiar el pasado para comprender la configuración del presente, mediante formas específicas de reflexión, eran tareas propuestas por la Escuela Serena. Marcas de esta labor han quedado en los cuadernos de clase conservados en el Archivo Cossettini como así también el los diarios de la maestras y en fotografías. El registro constante de estas docentes es lo que permite pensar cómo se instrumentalizó la propuesta del Plan Asociado.

\section{El contenido de la forma: el Plan Asociado de la Escuela Serena}

El perfil del diseño curricular de la escuela Carrasco fue claramente interdisciplinario. Sin embargo, el sentido de lo interdisciplinar no estuvo presente en el léxico de las educadoras. Ellas propusieron un Plan Asociado de conocimientos concretos. ${ }^{5}$ En ese plan, sin perder la identidad disciplinar, los contenidos fueron articulados en una tabla de doble entrada. El plan quedó articulado en grados (vertical) y por contenidos, actividades y excursiones (horizontal). Los saberes específicos identificados fueron: geografía, historia, economía, sociedad, cívica e higiene. Así, las docentes tenían el panorama relacional de los contenidos a trabajar con los diferentes grupos de alumnos. Asociar era integrar, combinar, compatibilizar conceptos, principios y hechos a los efectos de que los educandos aprendieran como en la vida misma. Esto es, actuando y aplicando los distintos saberes en función de los avatares presentados en el día a día. La originalidad de este Plan residió no solo en el esfuerzo de articulación, sino en la prescripción de una serie de excursiones y actividades fuera de la escuela en las cuales los niños debían aplicar o en otras situaciones construir los saberes sugeridos en la escuela. Estas actividades fueron: visitar vecinos, conocer y respetar las señales viales, visitar negocios del barrio, recorrer el barrio, observar y estudiar el río, entrevistar a los vecinos, ayudar en situaciones hogareñas cotidianas, involucrarse en la vida productiva del entorno, cultivar la huerta, cuidar el jardín, intervenir en la naturaleza, etc.

Ahora bien, la novedad con respecto a la educación tradicional de corte normalista no así para la escolanovista, fue enunciar los contenidos en términos de Ciencias Sociales y, paso seguido, desagregar dentro de estas, las especificidades que permiten entender lo social. Allí estuvo presente la historia. A continuación, en la Tabla 1, transcribimos la selección de temas para trabajar en las clases de historia.

5 Los originales de dicho plan se encuentran en el ARCHIVO COSSETTINI perteneciente al Consejo Nacional de Investigaciones Científicas y Tecnológicas y sito en el Instituto Rosario de Investigaciones en Ciencias de la Educación, de la ciudad de Rosario. Los hemos consultado ahí y posteriormente en la obra de Marcela Pelanda, quien realizó un trabajo heurístico sobre el mismo. Es decir lo transcribió, digitalizó y publicó en: Marcela Pelanda (1995). 


\begin{tabular}{|c|c|}
\hline \multicolumn{2}{|r|}{ La historia en el currículum de la Escuela Serena (1935-1942) } \\
\hline $\begin{array}{l}\text { Año/ } \\
\text { nivel }\end{array}$ & Contenidos \\
\hline Jardín & $\begin{array}{l}\text { La bandera. El } 25 \text { de mayo. } \\
\text { Relatos históricos. } \\
\text { Anécdotas de Belgrano y Sarmiento. } \\
\text { Historia de la familia, de la escuela. }\end{array}$ \\
\hline${ }_{\text {inferior }}{ }^{\circ}$ & $\begin{array}{l}\text { La bandera, la escarapela, Belgrano. } \\
\text { El himno nacional. } \\
\text { El } 25 \text { de mayo, el } 9 \text { de julio. San Martín, Sarmiento. } \\
\text { El día de las Américas. } \\
\text { El origen del barrio. }\end{array}$ \\
\hline $\begin{array}{r}1 .{ }^{\circ} \\
\text { superior }\end{array}$ & $\begin{array}{l}\text { Historia de la escuela. Identidad del barrio. Visita a un viejo poblador. Ayer y } \\
\text { hoy. Casas viejas y casas nuevas. } \\
\text { Historia del alumbrado. Visitas al pescador, herrero, correo, policía, guardián } \\
\text { de la plaza. } \\
\text { La revolución de mayo. Moreno. costumbres de la época. El } 9 \text { de julio. San } \\
\text { Martín y la libertad. Sarmiento en la educación argentina. }\end{array}$ \\
\hline $2 .^{\circ}$ & $\begin{array}{l}\text { El barrio. Su historia. Rosario. Fundación o fortín. } \\
\text { Aldea, villa, puerto, ciudad. } \\
\text { Pobladores: indios, colonos, ciudadanos. } \\
\text { Primeras viviendas: evolución. } \\
\text { Primeros transportes evolución. } \\
\text { Primeras industrias y cultivos. } \\
\text { Viajes de Colón, Solís, Magallanes y Gaboto. } \\
\text { La época colonial, costumbres. } \\
\text { Revolución de mayo. Ideas de libertad, Belgrano. } \\
\text { El congreso de } 1816 \text {. San Martín en San Lorenzo, su ideal de libertad. } \\
\text { Rivadavia. Sarmiento maestro. }\end{array}$ \\
\hline $3 .^{\circ}$ & $\begin{array}{l}\text { Importancia histórica del Paraná en la conquista colonial e independencia. El } \\
\text { pasaje o juramento. El Carcarañá, Fuertes del S. Spíritu. Garay abre las puertas } \\
\text { a las tierras. } \\
\text { Primitivos habitantes. Vida. Costumbres. } \\
\text { El indio, carácter. La lucha de los Conquistadores con el indio. Exploradores, } \\
\text { conquistadores y colonizadores. Vida de los primeros fundadores. Fundación de } \\
\text { Santa Fe de la Veracruz. Santa Fe posta y punto de enlace de Buenos Aires y } \\
\text { Perú. Colonizadores. La conquista gringa. } \\
\text { Fundación de Esperanza, Coronda, San Carlos, Rafaela. } \\
\text { Inmigración. Rivadavia. Sarmiento y Alberdi en la inmigración. Santa Fe } \\
\text { y la revolución de mayo. Santa Fe y el Congreso de Tucumán. Santa Fe y la } \\
\text { constitución nacional. } \\
\text { Estanislao López, defensor de la autonomía provincial. }\end{array}$ \\
\hline
\end{tabular}




\begin{tabular}{|c|c|}
\hline $4^{\circ}$ & $\begin{array}{l}\text { Conquista del continente americano. Fines. Los ríos, caminos de la unidad } \\
\text { continental. Continuidades de ideas. } \\
\text { Cómo desde la cabaña al pueblo se construyó la nación. Belgrano y la } \\
\text { libertad de comercio. Propulsor de la agricultura. Moreno. Representación de los } \\
\text { hacendados. El criollo y la lucha por la libertad. } \\
\text { Inmigración y colonización. Primeros inmigrantes. Fundación de colonias, } \\
\text { latifundios. Influencia de la inmigración en el progreso de Argentina y América. } \\
\text { Figuras típicas del campo de ayer y de hoy. } \\
\text { Rivadavia. Libertad individual. Su obra civilizadora. } \\
\text { Inmigración. Comercio. Moreno y la libertad de prensa. Artículo } 26 \text {. Asamblea } \\
\text { del año 13. Congreso de Tucumán. Ideal de independencia. San Martín. } \\
\text { Rosas. Urquiza. Libre navegación de los ríos. Libertad y despotismo. } \\
\text { Importancia de la constitución en la vida política de los pueblos. } \\
\text { Historia del alumbrado, del primer ferrocarril. Hombres argentinos y } \\
\text { extranjeros que han continuado el progreso. }\end{array}$ \\
\hline $5^{\circ}$ & $\begin{array}{l}\text { Países de mayor influencia en el mundo conocidos antes del descubrimiento. } \\
\text { América precolombina. } \\
\text { El papel, la brújula y la imprenta ensanchaban el mundo conocido. } \\
\text { Colón, Vasco de Gama, Magallanes. Conquista de América. } \\
\text { Colonización, fundaciones. } \\
\text { Centros políticos, económicos y culturales más importantes de América } \\
\text { Colonial. } \\
\text { Antecedentes de los movimientos emancipadores en América. } \\
\text { Movimiento de la conciencia nacional revolucionaria. } \\
\text { Revolución de mayo. Obra de Moreno. Influencia de la Revolución de América } \\
\text { del Sur. Asamblea del año XIII. Obras. Gobiernos que le suceden. Independencia } \\
\text { de las colonias hispanoamericanas. } \\
\text { Rivadavia y su obra de gobierno. } \\
\text { La dictadura 1829-1852. Dictaduras del siglo XX: 1920-1944. } \\
\text { Urquiza y la reorganización nacional. Alberdi, Mitre, Sarmiento, Avellaneda, } \\
\text { Roca: constructores de una nueva República. }\end{array}$ \\
\hline $6 .{ }^{\circ}$ & Panamericanismo. \\
\hline
\end{tabular}

Tabla 1. Fragmento del Plan Asociado tomado de Pelanda (1995, pp: s/n)

La interpretación del listado de contenidos mostrado en la Tabla 1 abre los siguientes nudos de discusión. Primero, en cada grado la historia a enseñar oscila entre lo local (barrio en mayor medida, provincia en menor) y lo nacional (revolución, independencia, etc.). Se pasa de trabajar problemas específicos del barrio a plantear cuestiones vinculadas con la historia nacional. Lo que el proyecto no deja claro (con excepción de una nota sugerida para el $3^{\circ}$ grado donde se plantea Santa Fe y la Revolución) es cómo integrar esos saberes que los estudiantes construyen en el barrio con la historia nacional de revoluciones, próceres, etc. Aquí, introducimos las notas que Elvira Scalona (2007) destaca al reflexionar sobre el lugar de la historia local en las aulas. En esta línea, pensamos que si bien a principios de siglo XX la historia enseñada fue la del Estado nacional argentino con sus dos momentos claves: 25 de mayo y 9 de julio (junto a las fechas alusivas a los grandes varones que allí intervinieron: San Martín y Belgrano fundamentalmente), la historia local 
estuvo presente en algunas experiencias. Lo hizo en dos sentidos: por un lado, como escenario de la historia nacional dando cuenta de la ruta de los héroes (en tal año pasado San Martín por este lugar, aquí se detuvo Belgrano..., etc.), por otro, se aplicó a la historia de la ciudad, pueblo o barrio las mismas herramientas y criterios que el positivismo empleó en la historia nacional. Esto es, una historia de los principales acontecimientos políticos, masculinos y oficiales del lugar. Pero, en Cossettini hay un sesgo romántico (de impronta rousseauniana) que las remite a buscar una historia del paisaje, entonces recuperan actores que van más allá de la política. En Cossettini aparecen los oficios, los vecinos, los trabajadores. Lo que sí destaca la experiencia es que la historia como ciencia recupera un saber que es verdad y que reside en la memoria de quienes lo vivieron. Es por ello que los alumnos salen al encuentro de los vecinos para interrogar y construir el pasado.

En segundo lugar, advertimos una escasa enunciación de fechas. La temporalidad generalmente está marcada con las nociones de "ayer y hoy" (en un plano comparativo). Las fechas mencionadas se corresponden con las efemérides nacionales clásicas de la historia política: 25 de mayo de 1810 (la Revolución de Mayo), 9 de julio de 1816 (la declaración de la Independencia). Entendemos que esta supresión de las fechas se asocia con los principios de la psicología del desarrollo que asigna grados de construcción de la inteligencia.

En tercer lugar, se percibe la presencia abundante de nombres propios ligados todos ellos a la historia nacional: Sarmiento, Roca, Avellaneda, San Martín, Moreno, Belgrano, Rivadavia ${ }^{6}$. Los varones de la política, el intelecto y la guerra. Esos nombres se reiteran en los diferentes años muchas veces puntualizando en los mismos aspectos. Aparece un nombre caro a la historia política santafecina: Estanislao López ${ }^{7}$, pero también son mencionados con rótulos colectivos: los inmigrantes, el indio y los actores del barrio (herrero, policía, etc.). El uso del singular para nominar a los indios indica la lectura clásica y etnocentrista sobre el tema (como si fuesen lo mismo). Esta idea se refuerza con el plural que nomina la inmigración (provienen de distintos países europeos).

Finalmente, las ausentes en esta historia son las mujeres. Ellas no están ni en el plano de los nombres propios ni entre inmigrantes, indios y, ni siquiera, en los oficios del barrio. Pese a que en la Escuela Carrasco trabajaron fundamentalmente mujeres, el rescate del paso femenino por la historia no fue contemplado. Esta es una nota que puede fundamentarse en el enfoque historiográfico de la época (político, acontecimental, narrativo y masculino) que no contempló a las mujeres como sujetos de historia.

La historia propuesta hace hincapié en el barrio e incorpora la lógica nacional sugerida por el calendario escolar. Se expresa un esfuerzo por acompañar a los alumnos en la apropiación del pasado. Es por eso que los relatos familiares, la historia del barrio, la visita a vecinos, está permanentemente puesta como la principal clave de apropiación de saberes. Sin dudas, el aprendizaje coordina los contenidos y no viceversa, como solía suceder en las propuestas pedagógicas tradicionales que marcan como eje exclusivo la historia nacional. En Cossettini hay próceres, hay historia nacional pero también hay una preocupación porque los alumnos se apropien de la historia del barrio.

\section{Reflexiones finales}

A partir de las reflexiones y de la propuesta curricular de Olga Cossettini entendemos que, ella y su equipo de trabajo, enfocaron la tarea de enseñar suponiendo que no se puede comprender la realidad social encerrando a los educandos en el interior de las escuelas. Así, animados por los

6 Merece una nota informativa la lectura crítica que en Argentina se ha dado en torno al uso de las efemérides y de los héroes en las escuelas. Para ello es importante consultar los trabajos de Laura Méndez (2005); Perla Zelmanovich (1997) o Ricardo Lesser (2004), entre otros.

7 Estanislao López es uno de los nombres que resuena rápidamente cuando transitamos la historia política de las provincias argentinas. Es un emblema de la historia política santafesina. Para recorrer una mirada crítica sobre su perfil consultar el artículo de Griselda Tarragó y Darío Barriera “¿Quién era López?” (2006). 
principios del escolanovismo, los niños salieron de la escuela y se mezclaron con la gente del barrio para conocer y poder explicar la cotidianeidad que los rodea.

El tono especial que el escolanovismo asigna a esta experiencia es lo que facilita el cruce entre barrio, experiencias de los vecinos y construcción cognitiva por parte de los alumnos. Los chicos recorren las calles, hablan con los vecinos y así, con esas "fuentes vivas", van conociendo el pasado de su terruño. El gesto se teje con elementos del positivismo que observa y objetiva pero también con otros del romanticismo micheletiano (que sale y entrevista y así encuentra el sentido de la historia). Empero, la historia oficial aparece: en los listados de contenidos a enseñar y en los actos patrios. Lo que prima es la historia del Estado y sus dos acontecimientos clave: revolución e independencia. Allí se celebra el ser argentino. Pero, las hermanas Cossettini llegan a esa historia a partir de un diálogo entre los vecinos y los estudiantes. Aquí la historia no es un saber dado y cerrado sino un trabajo colectivo entre los niños y la gente. No hay propiedades con sello de autor sobre el conocimiento histórico, por el contrario es una construcción desde abajo.

Ahora bien, al estar marcada por el escolanovismo, esta experiencia puntualiza en el aprendizaje transformando el modo de entender el saber científico. Se enseña la historia pensando en aquellos contenidos que los niños y las niñas pueden aprender y no en las discusiones del campo historiográfico. Entonces las fechas son reemplazadas por las nociones de "antes y después", aparecen los vecinos con sus memorias y relatos. De este modo, cuando la forma de la enseñanza es modificada, también lo es el contenido. Si en el campo de la didáctica la forma afecta al contenido, entonces el caso Cossettini es un ejemplo de ello. Si quisiéramos buscar una lógica a la disposición de los contenidos, ella residiría en el aprendizaje y a partir de este dato se estructurarían las matrices de contenidos. Asimismo, como en esta experiencia particular se piensa en el niño y su capacidad de aprender haciendo en un lugar concreto, se plasman como tópicos a enseñar: la historia del barrio y su gente (lo más cercano). Probablemente, el análisis de esa historia se haya realizado en una clave positivista (la historia de campanario).

Esta propuesta estuvo acompañada no solamente de un cambio de estrategia pedagógica sino de una forma particular de entender la relación entre las ciencias en general. En el caso específico de la historia, las maestras entendieron que el disparador de las ciencias sociales y de la historia en particular es el medio geográfico. El ambiente, ese donde está situada la escuela, la casa, el barrio, el lugar donde trabajan los padres, la ciudad misma. Conocer el medio en términos empíricos (observando y preguntando a la gente) pero también teóricos (regresando a la escuela para reflexionar) fue la vía estimada para aprender.

Finalmente, la historia investigada y producida por los historiadores recibe sutiles transformaciones al entrar en las dinámicas de transmisión que genera la sociedad. La escuela fue y es uno de los espacios que se vale del discurso historiográfico no solo en las instancias curriculares sino en forma transversal. Por lo cual, es importante que los historiadores miremos qué sucede en el interior de estas instituciones, fundamentalmente porque es aquí donde, en forma masiva, los varones y mujeres tienen su primer encuentro con los relatos del pasado. En las escuelas la forma es contenido, el caso Cossettini da cuenta de ello. Al transformar el modo de enseñar, alteran el contenido de la historia, la vuelven local y localizada. En tanto dan protagonismo a la voz de los actores del barrio, los alumnos salen a buscar el saber histórico. Este gesto lejos de fundamentarse en términos historiográficos, lo hace en los propios de la pedagogía. Esto es, se aprende de lo cercano a lo lejano. Decisión pedagógica que altera el contenido de la historia a enseñar. Una historia que llega a la gente en forma masiva, que crece con ellos y que contribuye a solidificar un sentido común acerca del pasado. Así, es importante mirar qué sucede en las escuelas, cómo se presenta el conocimiento histórico que es materia de historiadores pero también es un saber clave para la construcción del lazo social, condición sine qua non para sostener la vida en sociedad. 


\section{Bibliografía}

Ansaldi, W. (1989). Soñar con Rousseau y despertar con Hobbes: una introducción al estudio de la formación del Estado nacional argentino. En Ansaldi, W., Moreno, J. (Org.), Estado y sociedad en el pensamiento nacional. Antología nacional para el pensamiento comparado (pp. 21-108). Buenos Aires: Cántaro.

Botana, N. (1994). El orden conservador. La política argentina entre 1880 y 1916. Buenos Aires: Sudamericana.

Bolufer. M. (2008). La vida y la escritura en el siglo XVIII. Inés Joyes: apología de las mujeres. Valencia: PUV.

Burke, P. (2003). Formas de hacer historia. Madrid: Alianza.

Caldo, P. y Garcilazo, R. (2016). ¿Volver a la escuela o abrir las puertas a ella? El desafío de explicar a los niños y adolescentes el oficio de historiar. Íber. Didáctica de las Ciencias Sociales, Geografía e Historia, 82, 62-66.

Carli, S. (2003). Niñez, pedagogía y política. Transformaciones de los discursos acerca de la infancia en la historia de la educación argentina entre 1880 y 1955. Buenos Aires: Miño y Dávila Editores.

Caruso, M. (2001). ¿Una nave sin puerto definitivo? Antecedentes, tendencias e interpretaciones alrededor del movimiento de la Escuela Nueva". En Pineau, P. et.al. (coord.), La escuela como máquina de educar. Tres escritos sobre un proyecto de la modernidad (pp. 93-134). Buenos Aires: Paidós.

Chartier, R. (2005). Pluma de ganso, libro de letras, ojo viajero. México: UIDH.

Chartier, R. (1999). Cultura escrita, literatura e historia. Conversaciones con Roger Chartier. México: Fondo de Cultura Económica.

Colotta, P. (2002). Escritura y poder en la Escuela Nueva argentina. En Cucuzza, H. Pineau, P. (dir.), Para una historia de la enseñanza de la lectura y la escritura en Argentina. Del catecismo colonial a la Razón de mi vida (pp. 253-274). Buenos Aires: Miño y Dávila.

Cossettini, O. y Cossettini, L. (2001). Obras completas. Rosario: Ediciones Amsafe.

Cucuzza, H. R. (2007). Yo Argentino. La construcción de la nación en los libros escolares (18731930). Buenos Aires: Miño y Davila.

De Amézola, G. (2008). Esquizohistoria. La historia que se enseña en la escuela, la que preocupa a los historiadores y una renovación posible de la historia escolar. Buenos Aires: El Zorzal.

Del Pozo Andrés, M. (2013). Justa Freire o la pasión de educar. Biografía de una maestra atrapada en la historia de España (1896-1965). Barcelona: Octaedro.

Eley, G. (2008). Una línea torcida. De la historia cultural a la historia de la sociedad. Valencia: PUV.

Fernández, S. y Caldo, P. (2013). La maestra y el museo. Gestión cultural y espacio público 19391942. Rosario: El Ombú Bonsái.

Finocchio, S. (2013). Nación: ¿algo para recordar?, ¿algo para celebrar?, ¿algo para proyectar? En Siade, I. (coord.), Ciencias Sociales en la escuela. Criterios y propuestas para la enseñanza (pp. 49-61). Buenos Aires: Aique.

González Bernaldo de Quirós, P. (2014). El largo siglo XIX. En Yankelevich, P. (coord.), Historia mínima de la Argentina (pp. 143-233). México: El Colegio de México.

González Delgado, M. (2013). Una aproximación historiográfica sobre el origen de los estudios de la caja negra. Las investigaciones del aula desde la historia de la educación en Gran Bretaña y Estados Unidos". Revista del currículum y formación del profesorado, 17, 390-404.

Guha, R. (2002). Las voces de la historia y otros estudios subalternos. Barcelona: Crítica.

Lesser, R. (2004). La infancia de los próceres. Belgrano, Rivadavia, Moreno, Castelli, Azcuénaga. Buenos Aires: Biblos.

Lobato, M. (2010). Nueva historia de la Argentina. El progreso, la modernización y sus límites 18801916, Tomo V. Buenos Aires: Sudamericana.

Méndez, L. (2005). Las efemérides en el aula. Aportes teóricos y propuestas didácticas innovadoras. 
Buenos Aires: Ediciones Novedades Educativas.

Ossanna, E. (1993). Una aproximación a la educación santafesina. En Puiggrós, A. (dir.), Historia de la educación en argentina. La educación en las provincias y territorios nacionales (18851945), Tomo IV (pp. 445-490). Buenos Aires: Galerna, 1993.

Pelanda, M. (1995) La escuela activa en Rosario. La experiencia de Olga Cossettini. Rosario: Irice. Pereyra, C. (1997). Historia para qué. En AAVV, Historia para qué (pp. 11-31). México: Siglo XXI.

Pérez, A. (2000). Navegar contra la corriente: la Ley de Educación Común, Normal y Especial, Santa Fe, 1934. Boletín de la Sociedad Argentina de Historia de la Educación, 2 época, 1, 47-54.

Romero, L. A. (2004). La Argentina en la escuela. La idea de nación en los textos escolares. Buenos Aires: Siglo veintiuno editores.

Scalona, E. (2007). La historia local como contenido de la enseñanza. En Fernández, S. (Comp.), Más allá del territorio. La historia regional y local como problema. Discusiones, Balances y proyecciones (pp. 169-178). Rosario: Prohistoria.

Tarragó, G. Barriera, D. (2006). Nueva Historia de Santa Fe IV. Adiós a la monarquía de los años revolucionarios a la crisis de 1820. Rosario: Prohistoria-La Capital.

Videla, O. (2006). Nueva Historia de Santa Fe. El siglo XX. Problemas sociales, políticas de Estado y economías regionales (1912-1976). Rosario: Prohistoria-La Capital.

Viñao Frago, A. (2008). La escuela y la escolaridad como objetos históricos. Facetas y problemas de la historia de la educación. História da Educaçao, 25, 9-54.

Zelmanovich, P. (1997). Efemérides entre el mito y la historia. Buenos Aires: Paidós.

Zemon Davis, N. (1998). ¿Quién es el dueño de la historia? La profesión del historiador. Entrepasados, 14, 111-118.

\section{Fuentes}

Jean-Jacques Rousseau. Emilio o de la Educación. Madrid: Edaf. 1985. [Prólogo de María del Carmen Iglesias y traducción de Luis Aguirre Prado]. 


\section{Panta Rei}

PANTA REI es una revista digital de investigación orientada a la Historia y otras ciencias afines. Su principal objetivo es la transmisión del conocimiento científico, dando una oportunidad también a los jóvenes investigadores que quieren abrirse camino en el estudio de las ciencias humanas y sociales. Se compone de estudios originales relacionados con la disciplina histórica así como su didáctica y difusión. Las diferentes secciones que componen la revista son: artículos de investigación, entrevistas a profesionales, recensiones de monografías de actualidad y crónicas de congresos o eventos científicos relevantes.

Todos los artículos publicados son objeto de un proceso de revisión a cargo de un mínimo de dos evaluadores, que se consideran expertos en el ámbito temático del artículo propuesto. Nuestro deseo es poder ofrecer unos contenidos rigurosos, de calidad y de interés.

EI CEPOAT (Centro de Estudios del Próximo Oriente y la Antigüedad Tardía de la Universidad de Murcia) es la institución encargada de la coordinación y gestión de la revista, desde donde anualmente se lanzará la convocatoria para aquellos que estén interesados en publicar sus trabajos, siempre relacionados con la Historia, Arqueología, Historia del Arte, Didáctica de las Ciencias Sociales, etc.

PANTA REI is a digital journal focused on History and other sciences related to it. Its main objective is the transmission of scientific knowledge by giving also an opportunity to young researchers who want to make their way in the study of human and social sciences. It is composed by original studies related to History, as well as its didactics and promotion. The different sections of this journal are: research articles, interviews to professionals, recensions on monographs about current issues and reports about congresses or relevant scientific events.

All the articles published are subject to a revision process carried out by a minimum of two reviewers who are considered to be experts in the field of the article proposed. Our wish is to offer rigorous contents with quality and being of interest to the reader.

CEPOAT (Centre of Studies of the Middle East and Late Antiquity of the University of Murcia) is the institution in charge of the coordination and management of this journal. This is the centre from where the call for papers will be launched for all the people interested in publishing their papers, always related to History, Archeology, Art History, Didactics of the Social Sciences, etc. 


\section{Normas de Publicación}

El autor se compromete a enviar trabajos originales, que no se encuentren publicados en otras revistas ni en otros idiomas. Así mismo, el mismo artículo no podrá ser presentado en otras revistas mientras dure el proceso de evaluación.

\section{Envío y presentación de originales}

Los artículos se enviarán exclusivamente a través del correo electrónico a la dirección pantarei@um.es. Los textos serán enviados en formato DOC y las imágenes en formato JPEG o TIFF, y con un tamaño mínimo de 2000 px. Éstas no aparecerán incorporadas en el texto, sino enviadas en archivo aparte y correctamente numeradas según su posición en el texto. Junto al trabajo, se rellenará y enviará un documento aparte en el que se especifiquen los datos del autor siguiendo el modelo disponible en la página Web de la revista.

Para la redacción de los trabajos se tendrá en cuenta el Manual de la American Psychological Association, en su sexta edición. La extensión máxima de los trabajos será de 30 páginas. La tipografía será Arial 11, con interlineado sencillo y sin espacio alguno entre párrafos. El texto deberá ir justificado a ambos márgenes y sin sangría en los primeros párrafos. Los márgenes serán de $2,50 \mathrm{~cm}$. En los casos en los que fuera necesario incorporar notas, éstas irán a pie de página, enumeradas consecutivamente, con tipografía Arial 10, interlineado sencillo y justificadas a ambos márgenes.

Una información más detallada se encuentra disponible en la página http://www.um.es/cepoat/ pantarei.

\section{Proceso de valoración y evaluación}

Una vez recibidos los trabajos, la Revista realizará una primera valoración. Si el trabajo enviado se ajusta a las normas de presentación propuestas, la temática es coincidente con la línea editorial de la revista y posee la calidad científica necesaria, será remitido al consejo asesor para una primera evaluación. Si no es así en este primer paso se puede rechazar directamente los documentos que incumplan claramente la línea editorial.

Será el Consejo Asesor quien indique a la revista la originalidad, relevancia, estructura, redacción, aparato bibliográfico, etc. del trabajo enviado y, para ello, se designará a dos revisores expertos externos que evaluarán cada uno de los trabajos, que pueden formar parte (o no) de este Consejo Asesor. La selección de los revisores se ajustará a la temática y características metodológicas del trabajo. El nombre y filiación de los autores serán eliminados del trabajo para su revisión, así como los revisores actuarán de manera anónima y confidencial.

Los revisores deberán rellenar un informe de evaluación que centrará su atención en aspectos tales como características formales, originalidad y novedad de los trabajos, relevancia de las propuestas y los resultados, calidad metodológica y validez científica.

Una vez terminado el proceso se decidirá la aceptación o no de los mismos y su publicación en el número que sea pertinente, así como las modificaciones susceptibles de ser realizadas para su final publicación. Dicha notificación se enviará únicamente por correo electrónico, en un plazo máximo de seis meses. 


\section{Publishing rules}

The author is committed to submit original papers not having been published in other reviews or in other languages. In this way, it is not allowed for the same paper to be presented in other reviews during the evaluation process.

\section{Submission and presentation of originals}

The articles will be exclusively submitted by email to pantarei@um.es. The texts will be submitted in DOC format and the images in JPEG or TIFF format, and with a minimum size of 2000 px. Images will not be integrated in the text but sent in another file and properly numbered according to their position in the text. Attached to the paper, a document will be filled out and sent where the author's data will be specified following the model available on the website.

The sixth edition of the Manual of the American Psychological Association will be taken into account for the writing of the papers. The length of the papers must not exceed 30 pages. Typography will be Arial 11 , with simple line spacing and no space between paragraphs. The text must be justified on both margins without indentation in the first paragraphs. Margins size will be $2.50 \mathrm{~cm}$. Where it could be necessary the incorporation of notes, they will be at the bottom of the page, consecutively numbered with typography Arial 10, simple line spacing and justified on both margins.

More detailed information is available on the website: http://www.um.es/cepoat/pantarei.

\section{Examination and assessment process}

The Journal will submit the papers to a first examination once received. If the paper follows the presentation guidelines, the subject agrees with the editorial line of this journal, and possess the scientific quality required, it will be sent to the advisory council for a first assessment. If not, the documents which clearly fail to complete the editorial line may be rejected straightaway in this first step.

The Advisory Council will indicate the originality, relevance, structure, writing, bibliography, etc. of the text to the journal; for this purpose, two outside experts will be designated to review the papers; these experts can be (or not) part of this Advisory Council. The selection of the experts will adjust to the subject and methodological characteristics of the paper. Name and affiliation of the author will be eliminated from the text for its review, in this way experts will act anonymously and confidentially.

The experts will fill out an assessment report which will focus on aspects such as formal characteristics, originality and novelty of the papers, relevance and results of the proposal, methodological quality and scientific validity.

Once the process is finished, the acceptance or not of the papers and its publication in the corresponding edition will be decided, as well as the modifications that may be done for its final publication. This notification will be sent by email within 6 months maximum. 


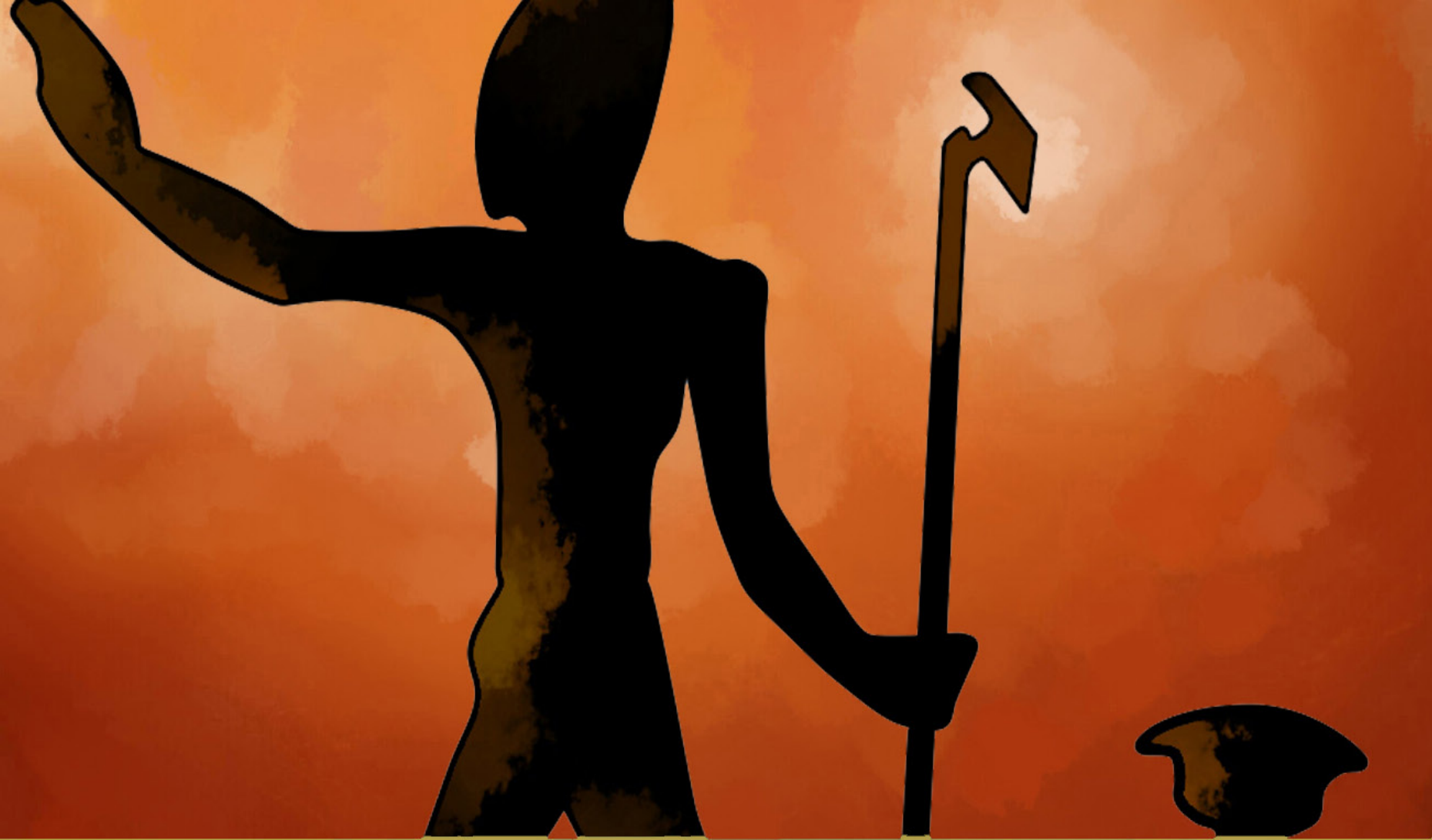

cepo t

UNIVERSIDAD DE MURCIA

centro de estudios del

próximo oriente y la

antigüedad tardía 